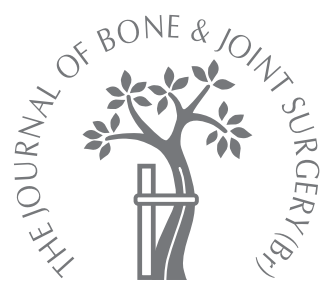

- CASE REPORT

\title{
Congenital meniscoid articular disc of the triangular fibrocartilage complex
}

S.-J. Kim,
H.-K. Moon,
Y.-M. Chun,
W.-H. Chang,
S.-G. Kim,
S.-K. Lee
From Yonsei
University College of
Medicine, Seoul,
Korea

Korea

\author{
We report the case of a 24-year-old man with a congenital meniscoid articular disc of the \\ triangular fibrocartilage complex with extensor carpi ulnaris tenosynovitis. His young age, \\ the normal articular cartilage, the lack of degenerative changes at the margins of the defect \\ and its bilateral occurrence made this diagnosis likely. A congenital defect of the articular \\ disc of the triangular fibrocartilage complex should not be misinterpreted as a traumatic \\ rupture and is usually asymptomatic.
}

Perforation of the triangular fibrocartilage complex resulting from injury or degenerative change are a well recognised source of ulnar wrist pain. ${ }^{1}$ Some authors have also raised the possibility of a congenital origin ${ }^{2-4}$ although others have opposed this view. ${ }^{5}$ Although there are a few studies reporting congenital perforations of articular disc ranging from slit to oval in shape, ${ }^{2,4}$ none has reported a meniscoid disc. We present such a case which is likely to be congenital and was demonstrated clearly by arthroscopic examination.

\section{Case report}

A 24-year-old man presented with a two-year history of ulnar wrist pain on his dominant side. He had been playing tennis, mainly at the weekends, from the age of 11 years but we did not consider this to be a significant factor. $\mathrm{He}$ described an intermittent snapping sensation of the wrist on supination beginning 1.5 years before consultation. Although it subsided following local steroid injections there remained some difficulty playing tennis and carrying heavy luggage. On examination, there was tenderness on the dorsi-ulnar aspect of the wrist but no swelling. The range of movement was normal. The grinding test ${ }^{6}$ showed mild pain without a click, the piano key test ${ }^{7}$ was negative and the ulnar impaction test caused some pain. Plain radiographs were unremarkable except for a $2 \mathrm{~mm}$ ulnar plus variance. MRI suggested a focal tear at the radial side of the articular disc (Fig. 1a) and tenosynovitis of extensor carpi ulnaris (Fig. 1b). Arthroscopic examination showed a meniscus-shaped triangular fibrocartilage complex (Fig. 2). The radial side of this complex was absent and the inner margin smooth and tapered. The dorsal and volar distal radio-ulnar ligaments appeared normal, which was consistent with the MRI findings. The central absence of the disc measured $6 \mathrm{~mm}$ in diameter and its appearance resembled the lateral meniscus in the knee. The articular cartilage appeared normal throughout. The disc showed no features of degeneration and the trampoline tension was intact. It was presumed the meniscoid disc might be congenital in nature and the synovitis of extensor carpi ulnaris could have been the source of the pain. MRI of the opposite, asymptomatic, wrist also showed a radial absence of the disc, similar to the right side. The pain had improved two years post-operatively, after conservative treatment including physiotherapy and non-steroidal anti-inflammatory drugs.

\section{Discussion}

The ulnar side of the wrist is an area of anatomical variation. ${ }^{8}$ A positive ulnar variance and the thickness of the articular disc have been proved to be negatively correlated..$^{9,10}$ Abe et $\mathrm{al}^{11}$ reported a case of a patient who had wrist pain with synovial proliferation due to triangular fibrocartilage complex thickening of $5.1 \mathrm{~mm}$ and $3 \mathrm{~mm}$ ulnar minus variance, whose pain was relieved by total resection of the triangular fibrocartilage complex. They mentioned that the thickened triangular fibrocartilage complex resembled the discoid meniscus of the knee, whereas our report is unique in that the configuration of the articular disc resembles a normal meniscus of the knee. 


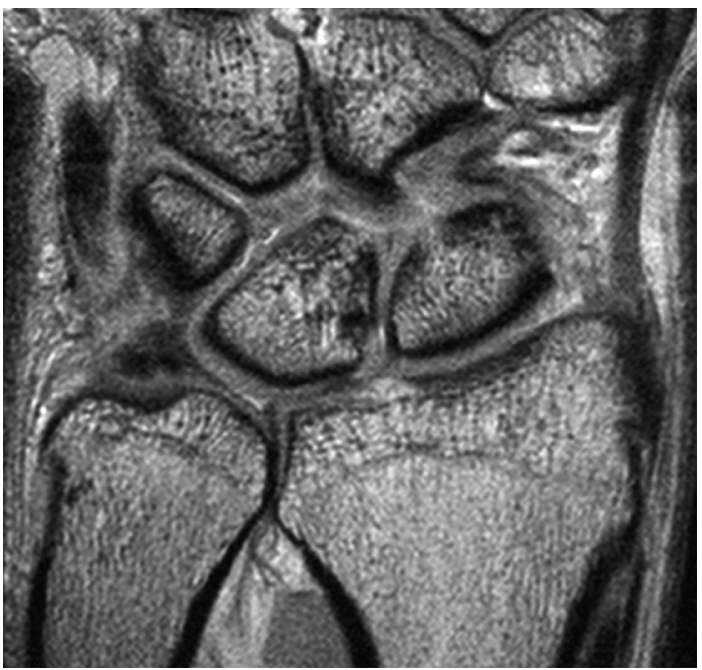

Fig. 1a

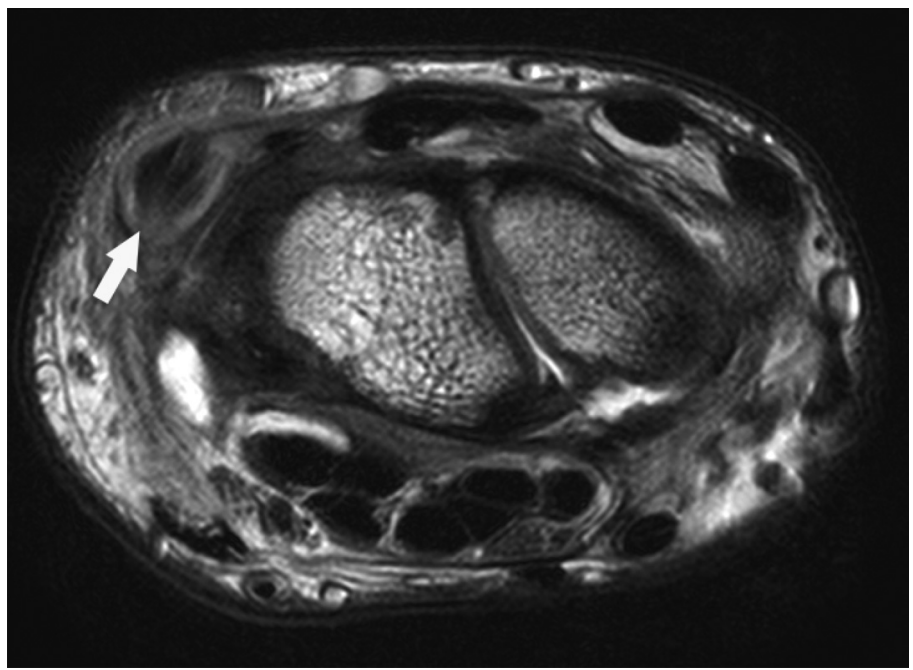

Fig. 1b

MRI of the wrist, a) proton density coronal view obtained at the mid-coronal section. The radial portion of the articular disc is not visible and a small amount of fluid is noted. The cartilage of the ulnar head and lunate are contacted with each other; and b) T2-weighted axial view at the level of proximal carpal row. Fluid collection involving the tendon sheath and tendinopathy of extensor carpi ulnaris tendon (white arrow) are compatible with tenosynovitis.

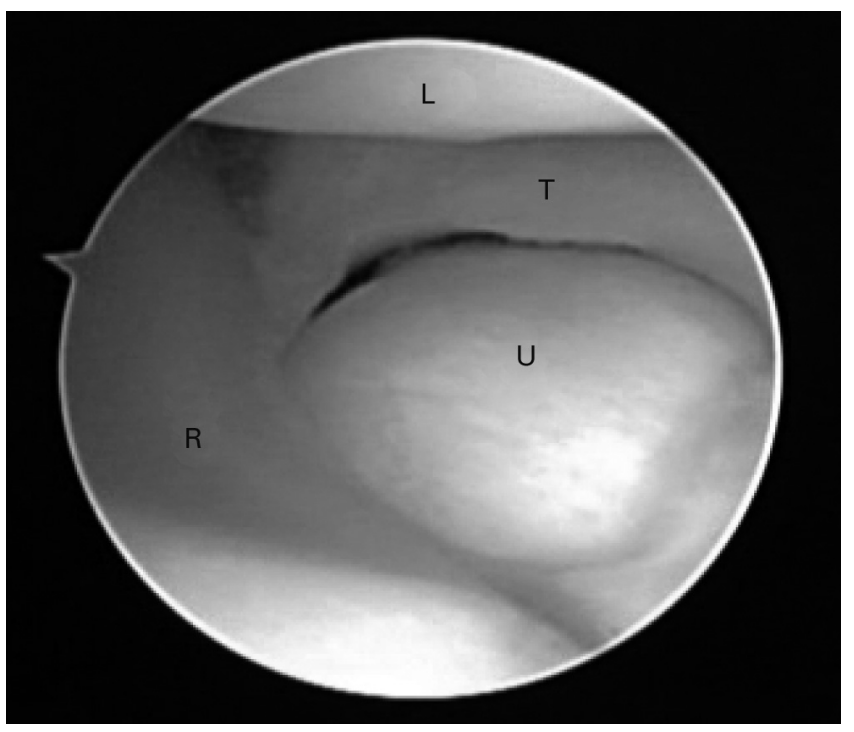

Fig. 2

Arthroscopic view through 3-4 portal showing a radial defect of the articular disc of the triangular fibrocartilage complex (3-4 represents the extensor compartments: 3 is the extensor pollicis longus and 4 is extensor digitorum communis, the 3-4 portal lies at the radiocarpal joint level between the extensor pollicis longus and extensor digitorum communis). The radial portion is absent and the ulnar margin is smooth and tapered. The dorsal and volar distal radio-ulnar ligament portion appears normal. The articular cartilage of the ulnar head, lunate and radius are normal $(R$, radius; $U$, ulnar head; $T$, triangular fibrocartilage complex; $L$, lunate)

In a cadaver study involving fetuses and infants, Tan et $\mathrm{al}^{2}$ demonstrated a high incidence $(26.7 \%)$ of congenital perforation of the triangular fibrocartilage complex all located between the radial attachment of the triangular fibrocartilage complex and the junction between its radial and middle third. The shapes and the site of the perforations were comparable with another study. ${ }^{4}$ There is only one in vivo case report of a possible congenital central perforation. ${ }^{3}$

Our case had a meniscoid configuration which may be considered a congenital anatomical variant. Traumatic or degenerative lesions are less likely causes, as the disc has a smooth and tapered margin and degenerative changes were not seen. It can also be argued that if the meniscoid lesion had been caused by repetitive stress, it would have been unilateral, whereas the asymptomatic contralateral wrist also showed a similar appearance. We therefore believe that the aetiology of the meniscoid configuration is congenital, and overuse while playing tennis combined with ulnar plus variance can explain the development of tendonitis of extensor carpi ulnaris. The disc itself does not require treatment.

The meniscoid articular disc is one of the congenital anatomical variants which can be over-diagnosed as a traumatic tear. Examination of the asymptomatic wrist with MRI may give valuable information regarding the cause of the lesion.

No benefits in any form have been received or will be received from a commercial party related directly or indirectly to the subject of this article.

\section{References}

1. Palmer AK. Triangular fibrocartilage complex lesions: a classification. J Hand Surg [Am] 1989; 14:594-606.

2. Tan AB, Tan SK, Yung SW, Wong MK, Kalinga M. Congenital perforations of the triangular fibrocartilage of the wrist. J Hand Surg [Br] 1995;20:342-5

3. Kaempffe FA. Central perforation of the articular disc of the triangular fibrocartilage complex in a 17-year-old-girl: could it be congenital? Am J Orthop 1997;26:565-7.

4. Kinninmonth AW, Chan KM. A study of age-related changes of the articular disc of the wrist in Hong Kong Chinese. J Hand Surg [Br] 1991;15:358-61. 
5. Chidgey LK, Dell PC, Bittar ES, Spanier SS. Histologic anatomy of the triangular fibrocartilage. J Hand Surg [Am]1991;16:1084-100.

6. Adams BD. Distal radioulnar joint instability. In: Green DP, Hotchkiss RN, Pederson WC, Wolfe SW, eds. Green's operative hand surgery. Fifth ed. Philadelphia: Churchill Livingstone, 2005:605-44.

7. Lichtman DM. Acute injuries of the distal radioulnar joint and triangular fibrocartilage complex. In: Ferlic DC, ed. Instructional course lectures. Vol. 52. Rosemont: American Academy of Orthopaedic Surgeons, 2003:177.
8. Hogikyan JV, Louis DS. Embryologic development and variations in the anatomy of the ulnocarpal ligamentous complex. J Hand Surg [Am] 1992;17:719-23.

9. Palmer AK, Glisson RR, Werner FW. Relationship between ulnar variance and triangular fibrocartilage complex thickness. J Hand Surg [Am] 1984;9:681-2.

10. Yoshioka H, Tanaka T, Ueno T, et al. Study of ulnar variance with high-resolution MRI: correlation with triangular fibrocartilage complex and cartilage of ulnar side of wrist. J Magn Reson Imaging 2007;26:714-19.

11. Abe Y, Kuwata N, Doi K, Kawai S. Wrist pain with triangular fibrocartilage thickening. Arch Orthop Trauma Surg 1999;119:98-9. 